\title{
Graph Theory-Guided Transcranial Magnetic Stimulation in Neurodegenerative Disorders
}

\author{
Ji Hyun Ko, ${ }^{1,2,3}$ Yoon Young Choi, ${ }^{1}$ and David Eidelberg ${ }^{1}$
}

${ }^{1}$ Center for Neurosciences, The Feinstein Institute for Medical Research, Manhasset, New York, United States of America;

${ }^{2}$ Department of Human Anatomy and Cell Science, University of Manitoba, Winnipeg, Manitoba, Canada; and ${ }^{3}$ Neuroscience

Research Program, Kleysen Institute for Advanced Medicine, Health Science Centre, Winnipeg, Manitoba, Canada

\begin{abstract}
The emergence of brain imaging techniques has shed light on the underlying mechanisms of brain diseases. Abnormalities found in the diseased brain have often been targeted by transcranial magnetic stimulation (TMS), which can modulate long-term neuronal excitability in humans in a noninvasive manner. Thus, its therapeutic application has been extensively explored for neurological and psychiatric disorders. As a result, TMS has been approved for clinical treatment for a few diseases, including drugrefractory depression. However, one of the biggest challenges with TMS is the difficulty in finding the optimal stimulation site. Until now, this process has been heavily dependent on previous activation studies and anatomical knowledge of the region itself, but it largely ignored the whole brain network that interacts with the focal brain region that has been targeted. Here, we propose a novel approach to estimate the prospective network effect following focal interference induced by TMS as a way to optimize the target identification process for TMS research.
\end{abstract}

Online address: www.bioelecmed.org

doi: 10.15424/bioelectronmed.2014.00004

\section{INTRODUCTION TO BRAIN IMAGING AND NETWORK ANALYSIS}

The development of brain imaging techniques, such as positron emission tomography (PET), single photon emission computed tomography (SPECT) and functional magnetic resonance imaging (fMRI), has greatly advanced our understanding of the human brain at the systems level. $\left[{ }^{18} \mathrm{~F}\right]$-fluorodeoxyglucose (FDG) is the most widely used PET radiotracer which can quantify glucose metabolism in neural tissue. It has been well documented that glucose metabolism correlates with neuronal activity (1); abnormal neuronal activity associated with neurological or psychiatric disorders may be identified using FDG PET. The most conventional approach for PET data analysis is statistical parametric mapping (SPM), which localizes the brain region that deviates from normal FDG uptake in control subjects $(2,3)$. The degree of this regional difference may be utilized as a biomarker for diseases $(4,5)$.

Multivariate analysis utilizes the covariances of whole-brain metabolic activity $(6,7)$. For example, the scaled subprofile mapping (SSM) - a form of principal component analysis (PCA) - has been used to analyze FDG PET scan data in patients with Parkinson's disease (PD). The resulting spatial covariance pattern, PD-related metabolic pattern (PDRP) $(8,9)$, significantly separates PD from the control group, and the network pattern

Address correspondence to David Eidelberg, Center for Neurosciences, The Feinstein Institute for Medical Research, 350 Community Drive, Manhasset, NY 11030. Phone: 516-5622498; Fax: 516-562-1008; E-mail: david1@nshs.edu.

Submitted August 6, 2014; Accepted for publication September 9, 2014; Published Online (www.bioelecmed.org) December 2, 2014.

\section{The Feinstein Institute for Medical Research Empowering Imagination. Pioneering Discovery.

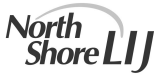

has been repeatedly validated in independent PD populations $[c f .(10,11)]$. One of the most advantageous features of multivariate analysis is that the degree of pattern expression can be quantified in each individual (12). The rest-state measurements of each subject's PDRP expression have been found to correlate consistently with clinical ratings of PD motor dysfunction and have been employed as a treatment biomarker in clinical trials of novel antiparkinsonian interventions (13). In addition, an automated metabolic imaging-based algorithm has demonstrated highly accurate results in differentiating idiopathic PD from other atypical parkinsonian syndromes, especially in the early stages of the disease (14). Notably, the prediction of accurate diagnosis was possible even before patients' motor symptoms emerged (15).

\section{TRANSCRANIAL MAGNETIC STIMULATION}

Transcranial magnetic stimulation (TMS) is a technique that has been used widely for noninvasive brain stimulation to examine motor, perceptual and cognitive processes (16-18). When applied 
continuously, repetitive TMS (rTMS) has been shown to produce profound and long-lasting effects on neuronal excitability. Therefore, when combined with neuroimaging techniques, one can assess the causality of the brain activity and behavior using the "perturb and measure" protocol [cf. (19)].

Traditionally, rTMS can be subdivided into fast $(>5 \mathrm{~Hz})$ and slow $(<1 \mathrm{~Hz})$, regarded as facilitatory and inhibitory, respectively (18). Interestingly, initial rTMS-PET studies reported increased regional cerebral blood flow (rCBF) at the stimulated site by both fast (20) and slow (21) rTMS. FDG uptake was also increased at the site of stimulation with intermediate frequency (2 Hz) (22). The unexpected increase in $\mathrm{rCBF}$ after slow (inhibitory) rTMS was explained by the active inhibition process $(23,24)$. A few studies, however, found reduced rCBF and FDG uptake at the stimulated site $(25,26)$ after slow rTMS.

The effect of rTMS in remote areas from the target site is more complicated. Recent literature suggests that the effect of rTMS can vary depending on the location, frequency, intensity, number of stimulation and the functional status of the target site or population (27). For example, the primary motor area (M1) is the most extensively investigated region in TMS-PET studies due to the large existing literature from neurophysiology (28). Majority of the TMS-PET studies reported increased $\mathrm{rCBF}$ at the stimulation site (20-22,29-33), although a few studies reported otherwise $(25,34)$. The remote effects of M1 rTMS has often involved the "motor network" [for review, see (28)]. For example, Lee et al. (35) proposed that $1 \mathrm{~Hz}$-rTMS applied to M1 induced a reorganization of the motor network by increasing the connectivity of the contralateral hemisphere to compensate for the inhibited M1 stimulation site. A similar approach was used to understand how patients with aphasia activate right-sided language function (36). Laird et al. (37) summarized the motor network that is influenced by M1 TMS using structured equation modeling and meta- analysis, and revealed that rTMS on M1 remotely changes activities in the supplementary motor area (SMA), anterior cingulate cortex (ACC), thalamus, premotor cortex (PMC), posterior parietal cortex and cerebellum. However, it should be noted that the direction of remote effect has not been consistent. For example, contralateral M1 activity was both increased $(33,38)$ and decreased $(21,30)$ after M1 rTMS.

The effect of rTMS on the prefrontal cortex (PFC) has not been consistent (33,39-42). Similar to M1 rTMS affecting motor network activities (37), PFC rTMS has modulated activities in the cognitive network comprised of ACC $(40,43)$, insula $(33,40)$, PMC $(41,42)$, basal ganglia $(33,40,43)$, parahippocampal region $(39,41,42)$ and cerebellum $(33,40)$. With respect to the clinical benefits, $\mathrm{rCBF}$ changes in the subgenual cingulate and orbitofrontal cortex after rTMS in the left dorsolateral prefrontal cortex have been reported previously to be associated with the efficacy of rTMS treatment in depression (44).

\section{TARGET IDENTIFICATION BY GRAPH THEORY}

While several studies investigated the effects of rTMS on neurodegenerative disorders, target identification has been a challenging issue. Most rTMS studies defined the target region based on activation studies [for example, $(45,46)$ ] and/or anatomical knowledge. The current practice largely ignores the prospective remote effects, although it has been clearly suggested that the whole-brain network is likely affected even if the stimulation was applied focally $(19,47)$. Here, we propose a novel research protocol for TMS target identification.

In the current study, we have applied a novel network analytical strategy based upon spatial covariance mapping $(48,49)$ and graph theory $(50,51)$ to identify the hub of a network that is associated with cognitive deficits in $\mathrm{PD}$, that is, $\mathrm{PD}$ cognitive pattern (PDCP) (52). It has been estimated that this hub region exhibits the greatest ability to modulate the expres- sion of the entire network during local stimulation. Because of the normalization inherent in the iterative eigenvector computations that we employ $(53,54)$, the loadings on the spatial covariance patterns do not vary sufficiently across regions for the delineation of discrete modules within the network space. That is, a different computational algorithm is necessary to define the organizational structure (topology) of the network after quantifying the relevant subject scores. To assess the flow of information within the PDCP, we used a graph theoretic approach (50). Adjacency matrices were constructed from interindividual correlation maps of 15 PD patients who were used in the derivation of PDCP (52). Automated anatomical labeling (AAL) was utilized to construct regions of interest (ROIs) for the whole brain regions (55). The resulting data matrix is both nonnegative and primitive, and is therefore assured based on the Perron-Frobenius theorem that it will have a single positive eigenvector. The component of this vector for a given ROI is called the eigenvector centrality (EC) for that region. EC represents a measure of information flow ranking each node according to its relative importance ("centrality") within the network space (56). Alternatively, in the context of a stochastic process, EC represents the likelihood of arriving at a given node after a long run of random walks irrespective of the starting point. The resulting data also can be used to deduce the organizational structure of an anatomical-functional network (for example, radial, linear, star-shaped, or core-periphery configurations).

The utility of graph theory in this context is demonstrated in a recent analysis of metabolic imaging data from PD patients $(n=99)$ and healthy volunteer subjects $(n=66)$ scanned in our laboratory (57). Extensive permutation testing of each group showed a strong relationship between the magnitude of the regional loadings (region weights) on the primary metabolic covariance pattern (the first principal component [PC]) and corresponding nodal EC values determined in- 
dependently by graph theory. Whether metabolically overactive (region weight $\mathrm{z}$ $>1.0)$ or underactive $(z<-1.0)$, the salient regions on the primary $\mathrm{PC}$ pattern can be viewed as major hubs of information flow along the graph theoretic network. Similarly, the regions with salient contributions to the PDCP topography functioned as information hubs along that network construct. This relationship was indicated by the presence of a significant correlation $(r=0.66, p<0.001)$ between regional loadings on the PDCP (representing the relative regional contributions to network activity) and the corresponding EC values (representing the relative importance of a given region to information flow along the network).

The sensitivity of each node to stimulation was quantified using EC derivative analysis (51) to identify regions that facilitate (or suppress) the influence of other "important" network hubs (defined as PDCP nodes with region weight $|z|>1$ ). For example, the right superior frontal gyrus (rSFG, BA 6/8) showed the highest EC derivative. Thus, altering the connectivity of this "sensitive" region by rTMS is likely to have the greatest influence on the centrality of the other salient PDCP nodes (that is, those with region weights).

By normalizing information flow within the PDCP network, therapeutic rSFG stimulation is expected to reverse the abnormal pattern expression that characterizes PD patients with cognitive dysfunction. The right middle frontal gyrus (rMFG, BA 8/9) has been viewed as the core of PD cognitive dysfunction (58-60) and thus chosen as the target of therapeutic rTMS in studies directed at this disease manifestation $(45,61)$. While this region exhibited greater importance (higher EC) than the rSFG, it had lower sensitivity, that is, an EC derivative value of relatively lower magnitude, making it comparatively less desirable as a treatment target.

\section{CONCLUSION}

We propose an innovative network targeting approach to optimize cortical
rTMS procedures to normalize aberrant brain networks, such as PDCP, which is associated with cognitive deficits in PD patients. To this end, disease-related network expression levels may be measured before and after rTMS is applied to one or more network nodes selected based on graph theoretic considerations.

\section{ACKNOWLEDGMENTS}

This work was supported in part by the National Institute of Neurological Disorders and Stroke Morris K Udall Center of Excellence for Parkinson's Disease Research at The Feinstein Institute for Medical Research (P50 NS071675 to D Eidelberg). The content is solely the responsibility of the authors and does not necessarily represent the official views of the National Institute of Neurological Disorders and Stroke or the National Institutes of Health. The sponsor did not play a role in study design, collection, analysis and interpretation of data, writing of the report or in the decision to submit the paper for publication.

\section{DISCLOSURE}

D Eidelberg serves on the scientific advisory boards of and has received honoraria from the Michael J Fox Foundation for Parkinson's Research and the Bachmann-Strauss Dystonia and Parkinson Foundation; is listed as a coinventor of patents named "Markers for Use in Screening Patients for Nervous System Dysfunction and a Method and Apparatus for Using Same" without financial gain; and has received research support from the NIH (NINDS, NIDCD, NIAID).

\section{REFERENCES}

1. Raichle ME, Mintun MA. (2006) Brain work and brain imaging. Annu. Rev. Neurosci. 29:449-76.

2. Friston KJ, Frith CD, Liddle PF, Frackowiak RS (1991) Comparing functional (PET) images: the assessment of significant change. J. Cereb. Blood Flow Metab. 11:690-9.

3. Friston KJ, et al. (1995) Statistical parametric maps in functional imaging: A general linear approach. Hum. Brain Mapp. 2:189-210.

4. Habeck CG. (2010) Basics of multivariate analysis in neuroimaging data. J. Vis. Exp. (41).

5. Habeck C, et al. (2008) Multivariate and univari- ate neuroimaging biomarkers of Alzheimer's disease. Neuroimage. 40:1503-15.

6. Habeck C, Stern Y, Alzheimer's Disease Neuroimaging Initiative. (2010) Multivariate data analysis for neuroimaging data: overview and application to Alzheimer's disease. Cell Biochem. Biophys. 58:53-67.

7. Niethammer M, Eidelberg D. (2012) Metabolic brain networks in translational neurology: concepts and applications. Ann Neurol. 72:635-47.

8. Ma Y, Tang C, Spetsieris PG, Dhawan V, Eidelberg D. (2007) Abnormal metabolic network activity in Parkinson's disease: test-retest reproducibility. J. Cereb. Blood Flow Metab. 27:597-605.

9. Eidelberg D, et al. (1994) The metabolic topography of parkinsonism. J. Cereb. Blood Flow Metab. 14:783-801.

10. Eidelberg D. (2009) Metabolic brain networks in neurodegenerative disorders: a functional imaging approach. Trends Neurosci. 32:548-57.

11. Ko JH, Spetsieris P, Ma Y, Dhawan V, Eidelberg D. (2014) Quantifying significance of topographical similarities of disease-related brain metabolic patterns. PLoS One. 9:e88119.

12. Spetsieris PG, Eidelberg D. (2011) Scaled subprofile modeling of resting state imaging data in Parkinson's disease: methodological issues. Neuroimage. 54:2899-914.

13. Feigin A, et al. (2007) Modulation of metabolic brain networks after subthalamic gene therapy for Parkinson's disease. Proc. Natl. Acad. Sci. U. S. A. 104:19559-64.

14. Tang CC, et al. (2010) Differential diagnosis of parkinsonism: a metabolic imaging study using pattern analysis. Lancet Neurol. 9:149-58.

15. Holtbernd F, et al. (2014) Abnormal metabolic network activity in REM sleep behavior disorder. Neurology. 82:620-7.

16. Pascual-Leone A, et al. (1998) Study and modulation of human cortical excitability with transcranial magnetic stimulation. J. Clin. Neurophysiol. 15:333-43.

17. Walsh V, Cowey A. (2000) Transcranial magnetic stimulation and cognitive neuroscience. Nat. Rev. Neurosci. 1:73-9.

18. Hallett M. (2007) Transcranial magnetic stimulation: a primer. Neuron. 55:187-99.

19. Ko JH, Strafella AP. (2012) Dopaminergic neurotransmission in the human brain: new lessons from perturbation and imaging. Neuroscientist. 18:149-68.

20. Paus T, et al. (1997) Transcranial magnetic stimulation during positron emission tomography: a new method for studying connectivity of the human cerebral cortex. J. Neurosci. 17:3178-84.

21. Fox P, et al. (1997) Imaging human intra-cerebral connectivity by PET during TMS. Neuroreport. 8:2787-91.

22. Siebner HR, et al. (1998) Imaging brain activation induced by long trains of repetitive transcranial magnetic stimulation. Neuroreport. 9:943-8.

23. Sohn YH, Wiltz K, Hallett M. (2002) Effect of volitional inhibition on cortical inhibitory mechanisms. J. Neurophysiol. 88:333-8. 
24. Waldvogel D, et al. (2000) The relative metabolic demand of inhibition and excitation. Nature. 406:995-8.

25. Paus T, et al. (1998) Dose-dependent reduction of cerebral blood flow during rapid-rate transcranial magnetic stimulation of the human sensorimotor cortex. J. Neurophysiol. 79:1102-7.

26. Valero-Cabre A, Payne BR, Pascual-Leone A. (2007) Opposite impact on 14C-2-deoxyglucose brain metabolism following patterns of high and low frequency repetitive transcranial magnetic stimulation in the posterior parietal cortex. Exp. Brain Res. 176:603-15.

27. Ziemann U, et al. (2008) Consensus: Motor cortex plasticity protocols. Brain Stimul. 1:164-82.

28. Reithler J, Peters JC, Sack AT. (2011) Multimodal transcranial magnetic stimulation: using concurrent neuroimaging to reveal the neural network dynamics of noninvasive brain stimulation. Prog. Neurobiol. 94:149-65.

29. Speer AM, et al. (2003) Intensity-dependent regional cerebral blood flow during $1-\mathrm{Hz}$ repetitive transcranial magnetic stimulation (rTMS) in healthy volunteers studied with $\mathrm{H} 215 \mathrm{O}$ positron emission tomography: I. Effects of primary motor cortex rTMS. Biol. Psychiatry. 54:818-25.

30. Fox PT, et al. (2006) Intensity modulation of TMSinduced cortical excitation: primary motor cortex. Hum. Brain Mapp. 27:478-87.

31. Rounis E, et al. (2005) Frequency specific changes in regional cerebral blood flow and motor system connectivity following rTMS to the primary motor cortex. Neuroimage. 26:164-76.

32. Siebner H, et al. (2001) Activation of frontal premotor areas during suprathreshold transcranial magnetic stimulation of the left primary sensorimotor cortex: a glucose metabolic PET study. Hum. Brain Mapp. 12:157-67.

33. Ferrarelli F, et al. (2004) A [17F]-fluoromethane PET/TMS study of effective connectivity. Brain Res. Bull. 64:103-13.

34. Siebner HR, et al. (2003) Patients with focal arm dystonia have increased sensitivity to slow-frequency repetitive TMS of the dorsal premotor cortex. Brain. 126:2710-25.

35. Lee L, et al. (2003) Acute remapping within the motor system induced by low-frequency repetitive transcranial magnetic stimulation. J. Neurosci. 23:5308-18.

36. Thiel A, et al. (2006) From the left to the right: How the brain compensates progressive loss of language function. Brain Lang. 98:57-65.

37. Laird AR, et al. (2008) Modeling motor connectivity using TMS/PET and structural equation modeling. Neuroimage. 41:424-36.

38. Siebner HR, et al. (2000) Lasting cortical activation after repetitive TMS of the motor cortex: a glucose metabolic study. Neurology. 54:956-63.

39. Paus T, Castro-Alamancos MA, Petrides M. (2001) Cortico-cortical connectivity of the human mid-dorsolateral frontal cortex and its modulation by repetitive transcranial magnetic stimulation. Eur. J. Neurosci. 14:1405-11.
40. Speer AM, et al. (2003) Intensity-dependent regional cerebral blood flow during $1-\mathrm{Hz}$ repetitive transcranial magnetic stimulation (rTMS) in healthy volunteers studied with $\mathrm{H} 215 \mathrm{O}$ positron emission tomography: II. Effects of prefrontal cortex rTMS. Biol. Psychiatry. 54:826-32.

41. Eisenegger C, Treyer V, Fehr E, Knoch D. (2008) Time-course of "off-line" prefrontal rTMS effects-a PET study. Neuroimage. 42:379-84.

42. Knoch D, et al. (2006) Lateralized and frequencydependent effects of prefrontal rTMS on regional cerebral blood flow. Neuroimage. 31:641-8.

43. Ohnishi T, et al. (2004) rCBF changes elicited by rTMS over DLPFC in humans. Suppl. Clin. Neurophysiol. 57:715-20.

44. Teneback CC, et al. (1999) Changes in prefrontal cortex and paralimbic activity in depression following two weeks of daily left prefrontal TMS. J. Neuropsychiatry Clin. Neurosci. 11:426-35.

45. Sedlackova S, Rektorova I, Srovnalova H, Rektor I. (2009) Effect of high frequency repetitive transcranial magnetic stimulation on reaction time, clinical features and cognitive functions in patients with Parkinson's disease. J. Neural Transm. 116:1093-101.

46. Srovnalova H, Marecek R, Rektorova I. (2011) The role of the inferior frontal gyri in cognitive processing of patients with Parkinson's disease: a pilot rTMS study. Mov. Disord. 26:1545-8.

47. Ko JH, Tang CC, Eidelberg D. (2013) Brain stimulation and functional imaging with $\mathrm{fMRI}$ and PET. Handb. Clin. Neurol. 116:77-95.

48. Spetsieris PG, Eidelberg D. (2011) Scaled subprofile modeling of resting state imaging data in Parkinson's disease: methodological issues. $\mathrm{Neu}$ roimage. 54:2899-914.

49. Spetsieris P, et al. (2013) Identification of diseaserelated spatial covariance patterns using neuroimaging data. J. Vis. Exp. (76).

50. Bullmore E, Sporns O. (2009) Complex brain networks: graph theoretical analysis of structural and functional systems. Nat. Rev. Neurosci. 10:186-98.

51. Correa C, Crnovrsanin T, Ma K. (2012) Visual reasoning about social networks using centrality sensitivity. IEEE Trans. Vis. Comput. Graph. 18:106-20.

52. Huang C, et al. (2007) Metabolic brain networks associated with cognitive function in Parkinson's disease. Neuroimage. 34:714-23.

53. Perron O. (1907) Zur Theorie der Matrices. Mathematische Annalen. 64:248-63.

54. Frobenius G. (1912) Ueber Matrizen aus nicht negativen Elementen. Sitzungsber Königl Preuss Akad Wiss. 456-77.

55. Tzourio-Mazoyer N, et al. (2002) Automated anatomical labeling of activations in SPM using a macroscopic anatomical parcellation of the MNI MRI single-subject brain. Neuroimage. 15:273-89.

56. Seneta E. (1981) Non-negative Matrices and Markov Chains. New York: Springer-Verlag.

57. Ko JH, Spetsieris P, Eidelberg D (2013) Efficiency and cost of PCA-derived Parkinson's disease- related metabolic covariance pattern. Poster session presented at: 19th Annual Meeting of the Organization for Human Brain Mapping; 2013 Jun 16-20; Seattle, WA.

58. Monchi O, et al. (2004) Neural bases of set-shifting deficits in Parkinson's disease. J. Neurosci. 24:702-10.

59. Ko JH, et al. (2013) Prefrontal dopaminergic receptor abnormalities and executive functions in Parkinson's disease. Hum. Brain Mapp. 34:1591-604.

60. Polito C, et al. (2012) Interaction of caudate dopamine depletion and brain metabolic changes with cognitive dysfunction in early Parkinson's disease. Neurobiol. Aging. 33:206.e29-39.

61. Boggio PS, et al. (2005) Effect of repetitive TMS and fluoxetine on cognitive function in patients with Parkinson's disease and concurrent depression. Mov. Disord. 20:1178-84.

Cite this article as: Ko JH, Choi YY, Eidelberg D. (2014) Graph theory-guided transcranial magnetic stimulation in neurodegenerative disorders. Bioelectron. Med. 1:15-8. 